University of Nebraska - Lincoln

DigitalCommons@University of Nebraska - Lincoln

Peter Dowben Publications

Research Papers in Physics and Astronomy

3-26-2007

\title{
The electronic band structure of $\mathrm{CoS}_{2}$
}

\author{
Ning Wu \\ University of Nebraska-Lincoln \\ Yaroslav B. Losovyj \\ University of Nebraska-Lincoln, ylozovyy@indiana.edu \\ David Wisbey \\ University of Nebraska-Lincoln \\ Kirill D. Belashchenko \\ University of Nebraska-Lincoln, belashchenko@unl.edu \\ M. Manno \\ University of Minnesota, Minneapolis, MN \\ See next page for additional authors
}

Follow this and additional works at: https://digitalcommons.unl.edu/physicsdowben

Part of the Physics Commons

Wu, Ning; Losovyj, Yaroslav B.; Wisbey, David; Belashchenko, Kirill D.; Manno, M.; Wang, L.; Leighton, C.; and Dowben, Peter A., "The electronic band structure of $\mathrm{CoS}_{2}$ " (2007). Peter Dowben Publications. 185. https://digitalcommons.unl.edu/physicsdowben/185

This Article is brought to you for free and open access by the Research Papers in Physics and Astronomy at DigitalCommons@University of Nebraska - Lincoln. It has been accepted for inclusion in Peter Dowben Publications by an authorized administrator of DigitalCommons@University of Nebraska - Lincoln. 


\section{Authors}

Ning Wu, Yaroslav B. Losovyj, David Wisbey, Kirill D. Belashchenko, M. Manno, L. Wang, C. Leighton, and Peter A. Dowben 
Published in Journal of Physics: Condensed Matter 19 (2007), 156224 (10 pp); doi:10.1088/09538984/19/15/156224 Online at http://stacks.iop.org/JPhysCM/19/156224 Copyright (C) 2007 IOP Publishing Ltd. Used by permission.

\title{
The electronic band structure of $\mathrm{CoS}_{2}$
}

\author{
Ning $\mathrm{Wu}^{1}$, Ya. B. Losovyj ${ }^{1,2}$, David Wisbey ${ }^{1}$, K. Belashchenko ${ }^{1}$, \\ M. Manno ${ }^{3}$, L. Wang ${ }^{3}$, C. Leighton ${ }^{3}$, and P. A. Dowben ${ }^{1,4}$ \\ ${ }^{1}$ Department of Physics and Astronomy and the Nebraska Center for Materials and Na- \\ noscience, University of Nebraska-Lincoln, Lincoln, NE 68588-0111, USA \\ ${ }^{2}$ Center for Advanced Microstructures and Devices, Louisiana State University, 6980 \\ Jefferson Highway, Baton Rouge, LA 70806, USA \\ ${ }^{3}$ Department of Chemical Engineering and Materials Science, University of Minnesota, \\ Minneapolis, MN 55455, USA \\ ${ }^{4}$ Corresponding author: Department of Physics and Astronomy, Behlen Laboratory of \\ Physics, University of Nebraska-Lincoln, Lincoln, NE 68588-0111, USA. Email: \\ pdowben@unlinfo.unl.edu
}

\begin{abstract}
Angle-resolved and energy-dependent photoemission was used to study the band structure of paramagnetic $\mathrm{CoS}_{2}$ from high-quality single-crystal samples. A strongly dispersing hybridized $\mathrm{Co}-\mathrm{S}$ band is identified along the $\Gamma-\mathrm{X}$ line. Fermi level crossings are also analyzed along this line, and the results are interpreted using band structure calculations. The Fermi level crossings are very sensitive to the separation in the S-S dimer, and it is suggested that the half-metallic gap in $\mathrm{CoS}_{2}$ may be controlled by the bondingantibonding splitting in this dimer, rather than by exchange splitting on the Co atoms.
\end{abstract}

\section{Introduction}

The experimental electronic structure of nominally ground-state half-metallic systems remains of considerable interest as a platform for studying the interplay between high spin polarization and band structure. This attraction remains in spite of the growing recognition that true half-metallic character may not be possible at finite temperatures $[1,2]$ due to magnons [1-5] as well as zero-temperature interactions [6,7]. Experimental data on band structure are available for very few nominally half-metallic systems, such as NiMnSb [8]. These studies have been largely limited by experimental difficulties in preparing a surface suitable for photoemission $[1,2]$.

The pyrite-type transition metal compound $\mathrm{CoS}_{2}$ is an itinerant electron ferromagnet. In ground-state band structure calculations [9-14], $\mathrm{CoS}_{2}$ is predicted to be highly spin polarized and at least close to being half-metallic, i.e. a ferromagnet with only one conducting spin channel. However, the measured saturation magnetization $\left(0.74 \mu_{\mathrm{B}} / \operatorname{CoS}_{2}\right.$ [15]; 
$0.85 \mu_{\mathrm{B}} / \mathrm{Co}[16] ; 0.87 \mu_{\mathrm{B}} / \mathrm{CoS}_{2}$ [17]) falls short of the expected $1 \mu_{\mathrm{B}} /$ Co for the hypothetical half-metallic $\mathrm{CoS}_{2}$. This means that the minority-spin valence states are present at the Fermi level, and $\mathrm{CoS}_{2}$ is not an ideal half-metallic ferromagnet. In this regard, $\mathrm{CoS}_{2}$ resembles the much touted $\mathrm{La}_{0.65} \mathrm{Sr}_{0.35} \mathrm{MnO}_{3}$ [18], although $\mathrm{CoS}_{2}$ is less complicated. $\mathrm{CoS}_{2}$ can be alloyed with the narrow band gap semiconductor $\mathrm{FeS}_{2}$ to become the highly spin-polarized ferromagnet $\mathrm{Fe}_{1-x} \mathrm{Co}_{x} \mathrm{~S}_{2}$, which more closely resembles an ideal ground-state half-metallic ferromagnet, by "tuning" the Fermi level $[12-14,19]$. The problem with $\mathrm{Fe}_{1-x} \mathrm{Co}_{x} \mathrm{~S}_{2}$ is that these systems will likely suffer from Co segregation [20], making the surface unstable, as is observed for many other nominally half-metallic systems $[1,2]$.

With an electron spin polarization of about $56 \%$, as determined from point-contact Andréev reflection [19], $\mathrm{CoS}_{2}$ remains highly spin polarized, with a Curie temperature in the range of $116-120 \mathrm{~K}[17,21]$, but not half-metallic. $\mathrm{CoS}_{2}$ is, nonetheless, a starting point for characterizing the electronic structure of the pyrite-type transition metal alloys. Understanding the $\mathrm{CoS}_{2}$ band structure is important, especially since the Fermi level crossings are very sensitive to the bonding-antibonding splitting in the sulfur dimer, as well as to the choice of the exchange-correlation potential $[10,11]$. In this paper, we demonstrate strong hybridization of cobalt and sulfur in the band structure, which is an essential ingredient for forming the high-polarization pyrite-type transition metal materials. The experimental bulk band structure, although spin integrated and taken at temperatures well above $T_{\mathfrak{c}}$, when compared with calculation suggests that $\mathrm{CoS}_{2}$ is not half-metallic. Analysis of the Fermi level crossings along the $\Gamma X$ line suggests that the half-metallic gap in the $\mathrm{CoS}_{2}$ ground state may be controlled by sulfur bonding-antibonding splitting, rather than by $\mathrm{Co}$ exchange splitting.

\section{Experimental details}

The success of this work was made possible by the cleavage of sufficiently large $\mathrm{CoS}_{2}$ (100) single crystals (several millimeters in diameter). The crystals were prepared by chemical vapor transport, and have well controlled stoichiometry, as detailed in a previous publication $[17,21]$. These crystals, when cleaved, provide low-energy electron diffraction (LEED) patterns characteristic of a highly ordered surface, as seen in figure 1(b). These LEED experiments were taken in the same ultrahigh-vacuum (UHV) chamber as the photoemission data, with a pressure of $1 \times 10^{-10}$ Torr. Surface composition and order are seen to be strongly dependent upon surface preparation, but the samples appear to be single crystals with no evidence of twinning or grain boundaries in the LEED or x-ray diffraction. Using the rocking curve x-ray diffraction data, we find that the lattice parameter is $5.508 \AA$. Sulfur segregation was easily obtained, but the results presented here are restricted to stoichiometric surfaces obtained by cleaving the crystals.

Angle-resolved photoemission spectra were obtained using plane-polarized synchrotron light dispersed by a $3 \mathrm{~m}$ toroidal grating monochromator at the Center for Microstructures and Devices (CAMD). The measurements were made in a UHV chamber employing a hemispherical electron analyzer with an angular acceptance of $\pm 1^{\circ}$, as described elsewhere [22]. The combined resolution of the electron energy analyzer and monochromator is $120-150 \mathrm{meV}$ for high kinetic photon energies $(50-120 \mathrm{eV})$, but higher resolution (about $80 \mathrm{meV}$ ) is obtained at lower photon energies of $25-40 \mathrm{eV}$. The photoemission experiments were undertaken with a light incidence angle of $45^{\circ}$ with respect to the surface normal and with the photoelectrons collected along the surface normal. All binding energies are referenced to the Fermi level, as determined from clean gold. The bulk band mapping was undertaken at room temperature, well above the Curie temperature. 

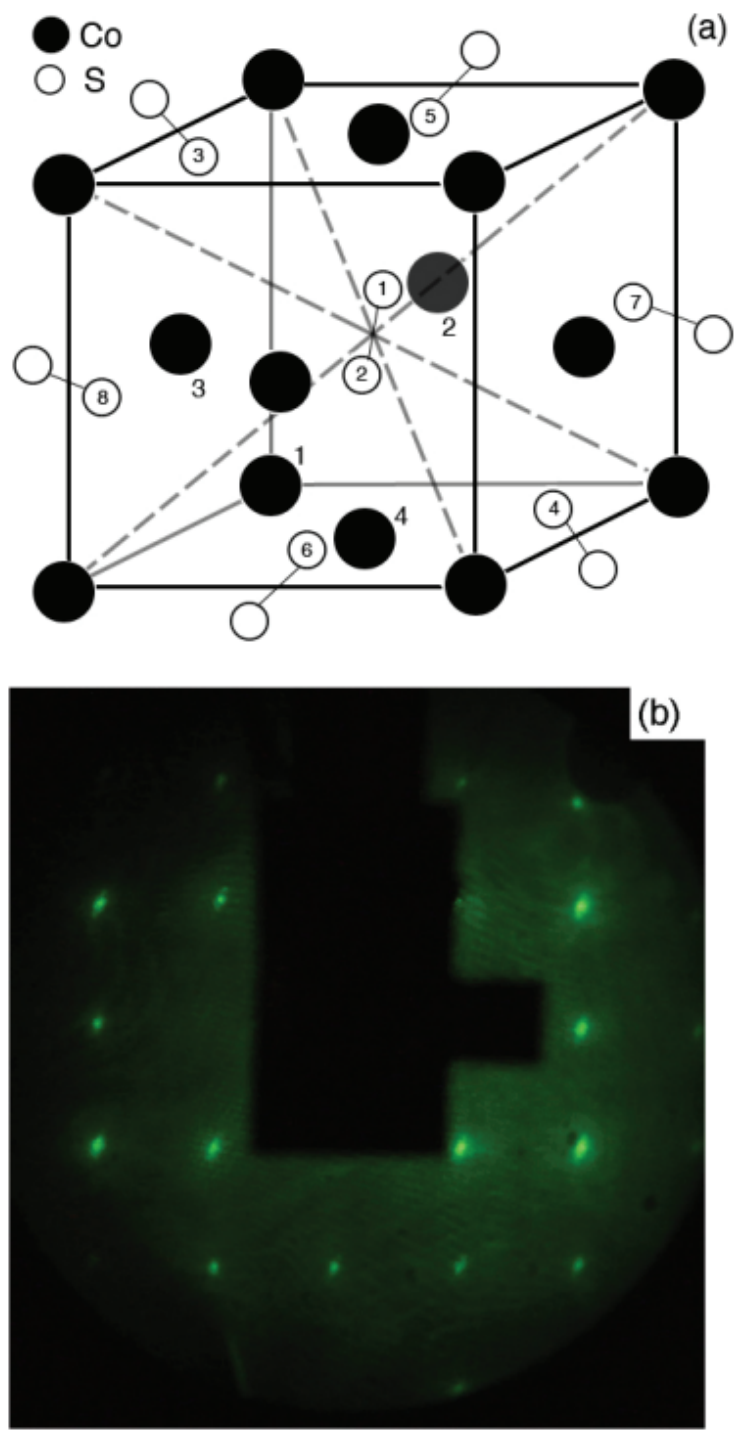

Figure 1. The schematic crystal structure for $\mathrm{CoS}_{2}$ is presented in (a). Due to its space group $\mathrm{T}_{\mathrm{h}}^{6}$, the cobalt atoms are located in an fcc cubic with position $(0,0,0),(0,1 / 2,1 / 2),(1 / 2,0$, $1 / 2)$ and $(1 / 2,1 / 2,0)$. The eight sulfur atoms are in the position $\pm(u, u, u ; u+1 / 2,1 / 2-u$, $\bar{u} ; \bar{u}, u+1 / 2,1 / 2-u ; 1 / 2-u, \bar{u}, u+1 / 2)$. The lattice constant $a_{0}$ is $5.524 \AA$ and $u$ is 0.389 , taken from Wyckoff [29]. (b) shows the low-energy electron diffraction (LEED) pattern for the highly ordered $\mathrm{CoS}_{2}(100)$ surface, taken with an incident electron energy $127 \mathrm{eV}$.

\section{Theory}

The calculations were performed for the ferromagnetic ground state on the assumption that $\mathrm{CoS}_{2}$ preserves some spin splitting up to room temperature. In other words, there is local magnetic order even well into the paramagnetic phase. This assumption is supported by ample experimental evidence:

(1) Magnetic susceptibility demonstrates Curie-Weiss behavior up to at least $1000 \mathrm{~K}$ [16]. 
(2) The photoemission measurements of [23] do not show any changes across the $\mathrm{Cu}$ rie temperature in the region attributed to the $\mathrm{Co}_{2 \mathrm{~g}}$ band. If the exchange splitting changed appreciably with temperature, the width of the Co $t_{2 g}$ band would change noticeably.

(3) Neutron diffraction measurements [24] show that the magnetic moment is preserved up to and above the room temperature.

(4) Very large electric resistivity was measured up to room temperature [17], which suggests a dominant spin-disorder contribution. Spin mixing due to spin disorder may broaden and shift the spin-split bands, but this analysis requires spin-resolved experimental data and is therefore left for future studies.

The electronic structure of $\mathrm{CoS}_{2}$ was calculated using the full-potential linear augmented plane-wave method (FLAPW) [25] implemented in the FLEUR code [26]. The generalized gradient approximation (GGA) was used for the exchange-correlation potential [27]. For the lattice parameter we took the value of $5.508 \AA$ obtained in this work. The internal structural parameter $x_{\mathrm{S}}$ (which determines the $\mathrm{S}-\mathrm{S}$ dimer separation) was varied in order to understand its effect on the band structure. The coupling of this structural parameter to magnetic order was observed earlier [28]. At the experimental value $x_{\mathrm{S}}=0.389[24,29]$ our band structure is essentially identical to the one obtained in [11] using the same method: the ground state is half-metallic, in disagreement with experiment [15-17, 19]. Contrary to GGA, the local density approximation (LDA) gives a ground state that falls short of being half-metallic, with minority-spin Fermi surface pockets around the R point [11]. It is clear that the inclusion of electronic correlations on the Co site should increase the exchange splitting, which would shift the minority-spin Co $\mathrm{e}_{\mathrm{g}}$ band upward and likely above the Fermi level, as happens already in GGA. Moreover, as we will see below, experimental data suggest the presence of at least one Fermi level crossing along the $\Gamma X$ line, which is absent either in LDA or in GGA, and is unlikely to appear if correlations were included. We therefore suggest that the disagreement with experiment may be due to a slight error either in the $\mathrm{S}-\mathrm{S}$ bonding-antibonding splitting or in the relative position of the $\mathrm{S} 3 \mathrm{p}$ and Co $3 \mathrm{~d}$ bands. To illustrate the consequences of this possible error, we have shown the band structure calculated for the decreased value $x_{\mathrm{S}}=0.387$, as discussed later. This shift increases the S-S distance by $0.038 \AA$ and the decreases the S- S bonding-antibonding splitting. We found that the value $x_{\mathrm{S}}=0.387$ corresponds to the minimum energy in the GGA approximation, which is in excellent agreement with the plane-wave pseudopotential calculation [28].

The calculated band structures along the $\Gamma \mathrm{X}$ line are shown in figure 2 (panels (a) and (b)). The size of the symbols shows the weight of the sulfur orbitals in the corresponding eigenstates. The band approaching the $\Gamma$ point close to the Fermi level has almost pure $\mathrm{S}$ Co weight, and is therefore almost unsplit by spin. Comparison of panels (a) and (b) shows that these sulfur bands notably shift down when the S-S separation is increased (as expected), and at $x_{\mathrm{S}}=0.387$ they cross the Fermi level in both spin channels. Thus, small errors in the position of the antibonding $\mathrm{S}-\mathrm{S}$ states may lead to the incorrect prediction of half-metallicity and the character of the minority-spin conduction band bottom in $\mathrm{CoS}_{2}$.

\section{The $\mathrm{CoS}_{2}$ bulk band structure}

Figure 3 illustrates the dependence of the normal emission photoemission spectra on photon energy from 16 to $31 \mathrm{eV}$. The broad photoemission features spread between -3 and -7 $\mathrm{eV}$ are ascribed to the $\mathrm{S} 3 \mathrm{p}$ bands. The sharp photoemission peak around $-0.8 \mathrm{eV}$ is attributed to the narrow, fully occupied bulk Co $3 \mathrm{~d}\left(\mathrm{t}_{2 \mathrm{~g}}\right)$ band. The partially occupied Co $3 \mathrm{~d}$ (bulk $\mathrm{e}_{\mathrm{g}}$ ) band can be seen as a shoulder to the prominent Co peak, near the Fermi level. 


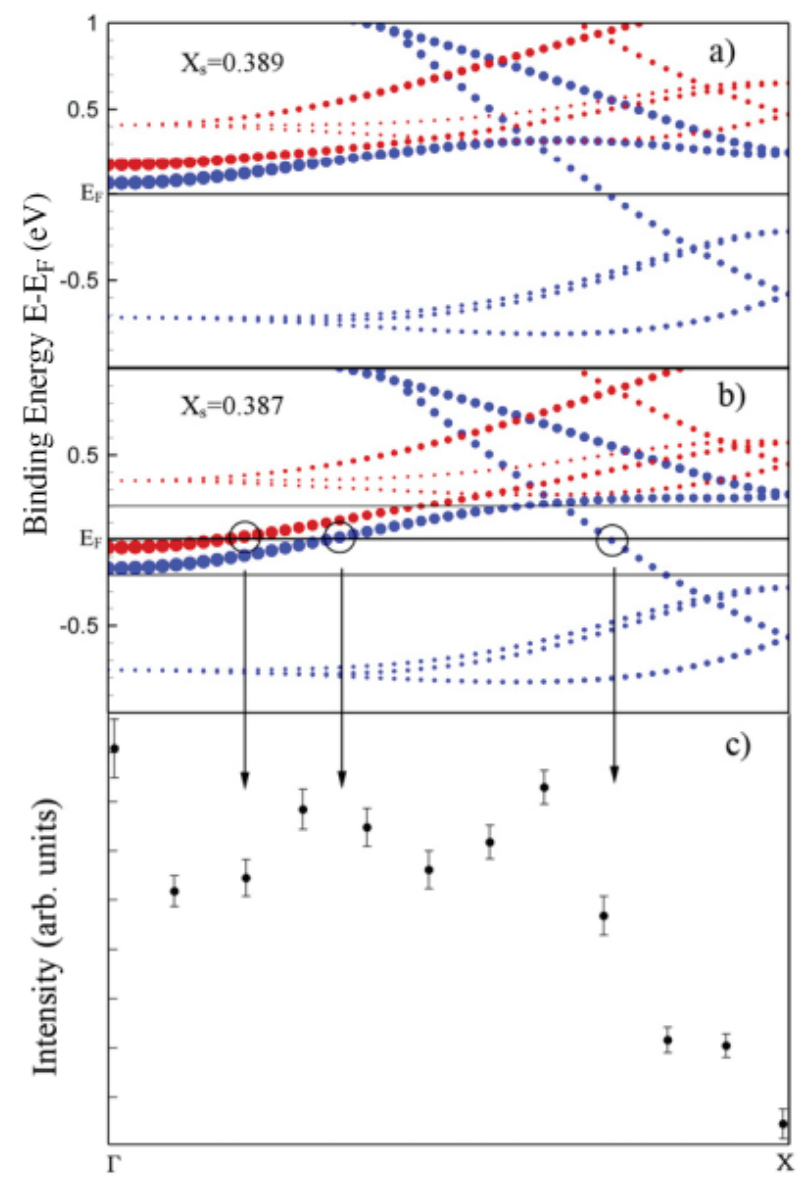

Figure 2. The intensity at the Fermi level, indicating the possible Fermi level crossings. The experimental intensity data (at bottom, i.e. (c)) are compared to theory for sulfur positions of $x_{\mathrm{S}}=0.389$ (a) and $x_{\mathrm{S}}=0.387$ (b). The blue and red curves are majority-and minority-spin bands, respectively. The expected band crossings of the Fermi level are indicated, and appear to agree with a sulfur position of $u=0.387$. The size of the symbols indicates sulfur weight in the corresponding eigenstates; the remaining weight belongs to $\mathrm{e}_{\mathrm{g}}$ states on the Co atoms.

High(er)-resolution photoemission spectra show that the Co $3 \mathrm{~d}\left(\mathrm{e}_{\mathrm{g}}\right)$ band is around -0.15 $\mathrm{eV}$ in binding energy, which is generally consistent with theory [9-11] and prior work [23]. The photoemission data of Takahashi and coworkers [23] also showed that the Co $3 \mathrm{~d}$ band can be resolved into two subbands in the vicinity of $1 \mathrm{eV}$ binding energy and at the Fermi level. Because of the changing cross-section with photon energy, at high photon energies near $40 \mathrm{eV}$ and above, two additional bands can be observed in the region of 3 and $9.5 \mathrm{eV}$ binding energies.

Although previous photon-energy-dependent photoemission has been undertaken [30, 31 ], the band structure was not as evident as in the case shown here (figure 3 ). This may be a consequence of the improved sample quality. Since the spectra presented in figure 3 are taken for normal emission, or $k_{\|}=0$, the peaks exhibiting photon energy dependence can be attributed to the bulk bands dispersing with $k_{\perp}$.

From the dispersion of the bands, evident in the photoemission spectra as a function of photon energy, we mapped the band structure along the ГX line. The perpendicular com- 


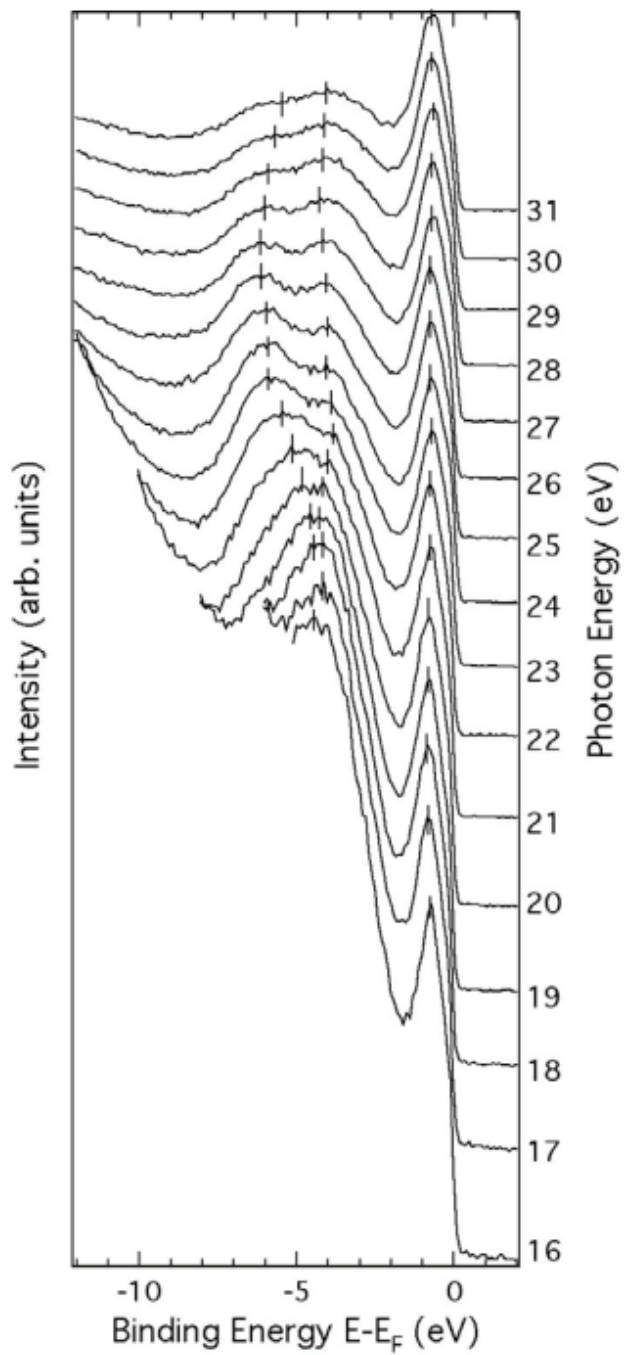

Figure 3. The photoemission spectra of $\mathrm{CoS}_{2}$ (100) for photon energies from 16 to $31 \mathrm{eV}$, taken at normal emission $\left(k_{\|}=0\right)$.

ponent of the crystal wavevector $k_{\perp}$ is not conserved across the crystal surface to vacuum interface, but instead can be found using the inner potential correction:

$$
k_{\perp}=\left[\frac{-2 m}{\hbar^{2}}\left\{E_{\mathrm{kin}}[\cos (\theta)]^{2}+U_{\mathrm{in}}\right\}\right]^{1 / 2}
$$

where $\theta$ is the emission angle of the photoelectron or the incident angle of the electron in inverse photoemission and $U_{\text {in }}$ is the inner potential of the solid. In most elemental metals the free-electron-like s band is well defined, and the inner potential $U_{\text {in }}$ is approximately equal to the difference between the vacuum level and the bottom of this free-electron-like $\mathrm{s}$ band $[32,33]$. However, this latter feature should not be expected to work for metallic compounds.

The experimental band structure is shown in figure 4 along the $\langle 100\rangle$ direction, or from $\Gamma$ to $\mathrm{X}$ of the bulk Brillouin zone. A photon energy of $27 \mathrm{eV}$, where the sulfur band 


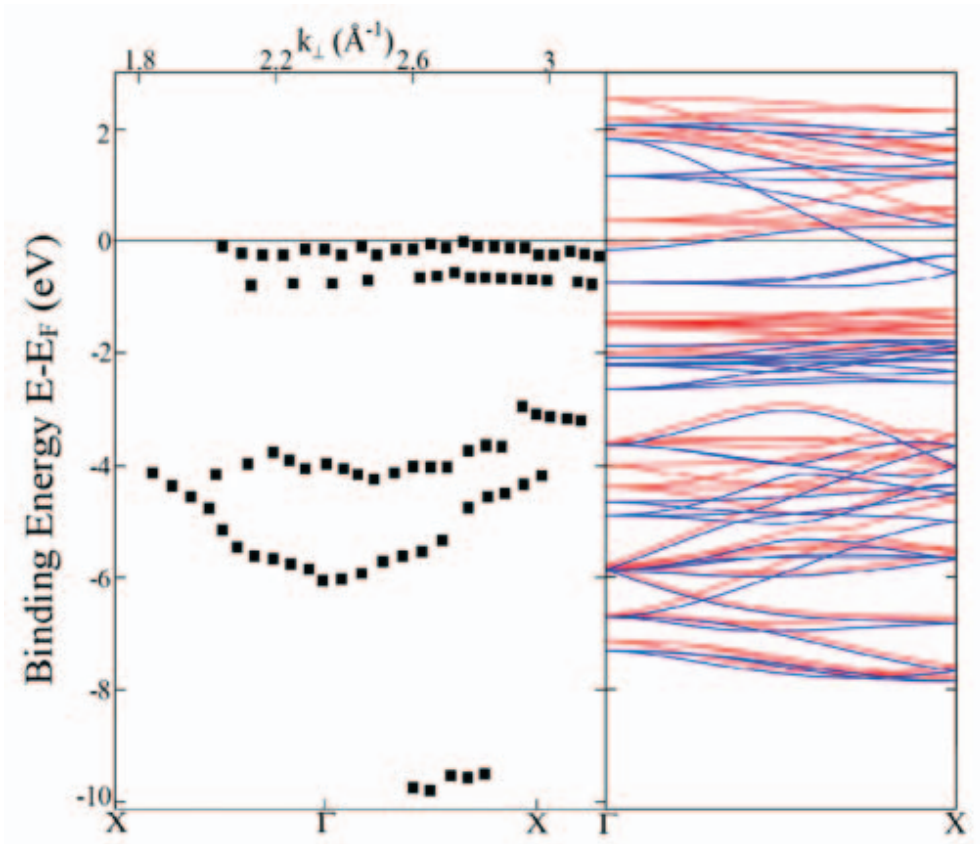

Figure 4. Experimental bulk band structure of $\mathrm{CoS}_{2}$ along the $\langle 100\rangle$ direction (left). The calculated bulk band structure for $x_{\mathrm{S}}=0.387$ (right). The blue and red curves are majority- and minority-spin bands, respectively. For the experimental data, both critical points and the experimental wavevectors are identified.

has the greatest binding energy, should correspond to the $\Gamma$ point in the $k$ vector space. The calculated bands along the $\Gamma \mathrm{X}$ line, shown in figures 2(b) and 4 for comparison, are qualitatively similar, although not all of the bands predicted by theory are clearly evident in experiment. The bands for two values of $x_{\mathrm{S}}$ are shown in figure 2 for reasons explained above in the theory section (section 3 ).

The strongly dispersing bands from 4 to $6 \mathrm{eV}$ binding energy have largely sulfur weight [9-11]. As in other theory calculations [9-11], our calculated Co $3 \mathrm{~d}$ bulk $\mathrm{t}_{2 \mathrm{~g}}$ band is located at a higher binding energy than indicated in experiment. The Co $3 \mathrm{~d}$ bulk bands, for both spin-up and spin-down states, are very flat (and with little dispersion) in both experiment and theory. Although all the Co $3 \mathrm{~d}$ bands show little evidence of dispersion, for different photon energies, we still believe the bulk $\mathrm{e}_{\mathrm{g}}$ band crosses the Fermi level based on the changes in the photoemission intensity at the Fermi level, shown in figure 2. As the photoemission intensities drop dramatically at about 0.2 and $0.4 \AA^{-1}$ either side of the $\Gamma$ point, we believe that there is an unresolved Fermi level crossing at about 2.1 and $2.5 \AA^{-1}$ and again at about $0.4 \AA^{-1}$ away from the $\Gamma$ point, in the $\operatorname{CoS}_{2}$ band structure (figure 2). The indications of the Fermi level crossing at $0.2 \AA^{-1}$ away from the $\Gamma$ point in the $\mathrm{CoS}_{2}$ band structure do not show the exchange split (spin-dependent) Fermi level crossings of theory (figure 2(b)). This could be because the small exchange splitting of the sulfur bands disappears at elevated temperatures, or because there is insufficient resolution to discern both bands predicted by theory. Further refinement of the experimental band structure, particularly near the Fermi level, is clearly indicated.

From the critical points of the experimental band structure, we can make an estimate of the inner potential. The $\Gamma$ critical point of $2.31 \AA^{-1}$ (in the second Brillouin zone) indi- 


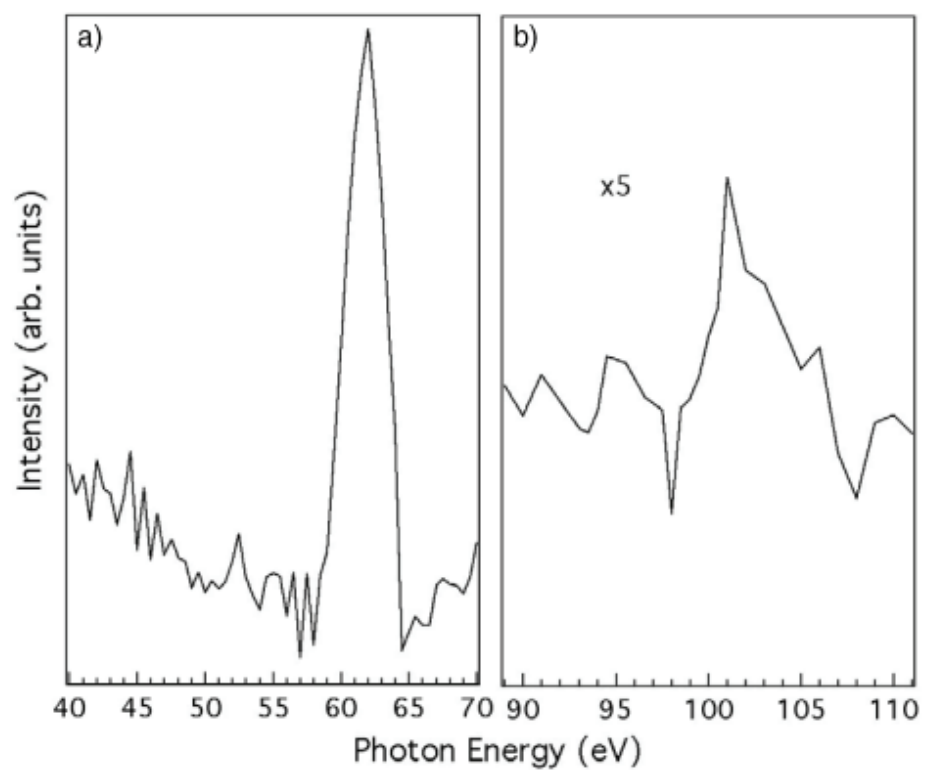

Figure 5. The photoemission intensities at $5 \mathrm{eV}$ binding energy with strong $\mathrm{S}$ weight, as a function of photon energy. The photoemission intensities are seen to increase at photon energies that are approximately at the Co 3p (a) and 3s (b) core edges, indicating strong hybridization of Co and $\mathrm{S}$ valence bands. The flux of synchrotron light in (b) amplified by factor 5 .

cates the small value of the inner potential, much smaller than in most transition metals (including $\mathrm{Ni}$ [34] and Mo [35]). The low value is not completely unreasonable, because in general there is no reason to expect the inner potential to be determined by the full bandwidth in a chemical compound.

\section{Cobalt-sulfur hybridization}

Theory suggests that the strongly dispersing bands at 4-6 eV binding energy, while containing strong sulfur contributions, also contain cobalt weight. To demonstrate that these valence band features do contain some cobalt contributions, resonant photoemission experiments were undertaken. We measured the photoelectron intensities of the weakly dispersing band from 4 to $5 \mathrm{eV}$ binding energy, as a function of photon energy, in $0.5 \mathrm{eV}$ increments. After flux normalization, the data of figure 5(a) show a sharp peak in the intensity of the largely sulfur initial state around $62 \mathrm{eV}$. This clear resonant photoemission enhancement occurs near the Co $3 \mathrm{p}$ shallow core edge around $59 \mathrm{eV}$, and is indicative of a possible super-Coster-Kronig resonance due to excitations involving the cobalt $3 p \rightarrow 3 d$ transition. The photoemission resonance is very narrow, indicating that the unoccupied Co $3 \mathrm{~d}$ bands are also very narrow, again consistent with theory $[10,11,23]$. We realize that the Co strongly hybridizes with S $3 p$ bands, as has been predicted $[11,36]$, through the relatively large-bandwidth Co $3 \mathrm{~d}\left(\mathrm{e}_{\mathrm{g}}\right)$ bands. According to theory, the $\mathrm{e}_{\mathrm{g}}$ band hybridizes with the $\mathrm{S} 3 \mathrm{p}$ and antibonding $3 \mathrm{p} \sigma^{*}$ unoccupied states [11]. The full width at half maximum of the photoemission resonance in figure 5(a) is approximately $3 \mathrm{eV}$, close to the calculated width of the $\mathrm{e}_{\mathrm{g}}-3 \mathrm{p}$ hybridized band and $3 \mathrm{p} \sigma^{*}$ bands [11]. Figure 5(b) indicates that a photoemission resonant enhancement occurs also due to the cobalt $3 \mathrm{~s} \rightarrow 4 \mathrm{p}$ transition, as the photoemission intensity increases at the Co $3 \mathrm{~s}$ core edge at $101 \mathrm{eV}$. This suggests that the 
$3 \mathrm{~s} \rightarrow 4 \mathrm{p}$ transition is much weaker compared with the $3 \mathrm{p} \rightarrow 3 \mathrm{~d}$ transition, as has been seen with transition metals [37].

\section{Summary}

From single crystals of $\mathrm{CoS}_{2}$, we have been able to map the experimental bulk electronic band structure along the $\Gamma \mathrm{X}$ direction. The $\mathrm{CoS}_{2}$ band structure near the Fermi level is sensitive to the $\mathrm{S}-\mathrm{S}$ separation, which suggests that the half-metallic gap may be controlled by antibonding $\mathrm{S} p$ rather than $\mathrm{Co}_{\mathrm{g}}$ states. Nonetheless, to sort out the correct band structure of the conduction band bottom, more detailed experimental data are needed. In addition, strong hybridization of cobalt and sulfur is indicated from the resonance photoemission at cobalt $3 p$ and 3 s core edges.

\section{Acknowledgments}

This research was supported through the US Office of Naval Research (ONR) grant no. N00014-06-1-0616, the Nebraska Research Initiative and the US National Science Foundation (NSF) "QSPINS" Materials Research Science and Engineering Center (DMR 0213808) at the University of Nebraska-Lincoln. The work at the University of Minnesota was supported by the NSF MRSEC under DMR-0212302. The Center for Advanced Microstructures and Devices is supported by the Louisiana Board of Regents.

\section{References}

[1] Dowben P A and Skomski R 2004 J. Appl. Phys. 95 7453-8

[2] Dowben P A and Jenkins S J 2005 Frontiers in Magnetic Materials ed A Narlikar (Berlin: Springer) pp 295-325

[3] Skomski R and Dowben P A 2002 Europhys. Lett. 58 544-8

[4] Itoh H, Ohsawa T and Inoue J 2000 Phys. Rev. Lett. 842501

[5] MacDonald A H, Jungwirth T, and Kasner M 1998 Phys. Rev. Lett. 81705

[6] Chioncel L, Katsnelson M I, de Groot R A, and Lichtenstein A I 2003 Phys. Rev. B 68144425

[7] Irkhin V Y and Katsnelson M I 1985 J. Phys. C: Solid State Phys. 184173

[8] Correa J S, Eibl C, Rangelov G, Braun J, and Donath M 2006 Phys. Rev. B 73125316

[9] Zhao G L, Callaway J, and Hayashibara M 1993 Phys. Rev. B 4815781

[10] Kwon S K, Youn S J, and Min B I 2000 Phys. Rev. B 62357

[11] Shishidou T, Freeman A J, and Asahi R 2001 Phys. Rev. B 64180401

[12] Mazin I I 2000 Appl. Phys. Lett. 773000

[13] Umemoto K, Wentzcovitch R M, Wang L, and Leighton C 2006 Phys. Status Solidi b 243 $2117-21$

[14] Ramesha K, Seshadri R, Ederer C, He T, and Subramanian M A 2004 Phys. Rev. B 70214409

[15] Ohsawa A, Yamaguchi Y, Watanabe H, and Itoh H 1976 J. Phys. Soc. Japan 40986

[16] Adachi K, Sato K, and Takeda M 1969 J. Phys. Soc. Japan 26631

[17] Wang L, Chen T Y, and Leighton C 2004 Phys. Rev. B 69094412

[18] Nadgorny B, Mazin I I, Osofsky M, Soulen R J, Broussard P, Stroud R M, Singh D J, Harris V G, Arsenov A, and Mukovskii Ya 2001 Phys. Rev. B 63184433 
[19] Wang L, Umemoto K, Wentzcovitch R M, Chen T Y, Chien C L, Checkelsky J G, Eckert J C, Dahlberg E D, and Leighton C 2005 Phys. Rev. Lett. 94056602

[20] Guevara J, Vildosola V, Milano J, and Llois A M 2004 Phys. Rev. B 69184422

[21] Wang L, Chen T Y, Chien C L, and Leighton C 2006 Appl. Phys. Lett. 88232509

[22] Waldfried C, McIlroy D N, and Dowben P A 1997 J. Phys.: Condens. Matter 910615

[23] Takahashi T, Naitoh Y, Sato T, Kamiyama T, Yamada K, Hiraka H, Endoh Y, Usuda M, and Hamada N 2001 Phys. Rev. B 63094415

[24] Brown P J, Neumann K-U, Simon A, Ueno F, and Ziebeck K R A 2005 J. Phys.: Condens. Matter 171583

[25] Weinert M, Wimmer E, and Freeman A J 1982 Phys. Rev. B 264571

[26] http://www.flapw.de

[27] Perdew J P, Burke K, and Ernzerhof M 1996 Phys. Rev. Lett. 773865

[28] Hobbs D and Hafner J 1999 J. Phys.: Condens. Matter 118197

[29] Wyckoff R W G 1965 Crystal Structures vol 1 (New York: Interscience)

[30] Fujimori A, Mamiya K, Mizokawa T, Miyadai T, Sekiguchi T, Takahashi H, Mori N, and Suga S 1996 Phys. Rev. B 5416329

[31] Muro T, Kimura A, Iwasaki T, Ueda S, Imada S, Matsushita T, Sekiyama A, Susaki T, Mamiya K, Harada T, Kanomata T, and Suga S 1998 J. Electron Spectrosc. Relat. Phenom. 88-91 361

[32] Plummer E W and Eberhardt W 1982 Adv. Chem. Phys. 49533

[33] Clarke L J 1985 Surface Crystallography: An Introduction to Low Energy Electron Diffraction (New York: Wiley)

[34] Eberhardt W and Plummer E W 1980 Phys. Rev. B 213245

[35] Yakovkin I N, Zhang J, and Dowben P A 2001 Phys. Rev. B 63115408

[36] Yamada H, Terao K, and Aoki M 1998 J. Magn. Magn. Mater. 177-181 607

[37] Sakisaka Y, Rhodin T N, and Dowben P A 1984 Solid State Commun. 49 563-5 